\title{
Generation of coherent terahertz pulses in Ruby at room temperature
}

\author{
Elena Kuznetsova ${ }^{1}$, Yuri Rostovtsev ${ }^{1}$, Nikolai G. Kalugin ${ }^{1}$ and \\ Roman Kolesov $^{1}$, Olga Kocharovskaya ${ }^{1}$, Marlan O. Scully ${ }^{1,2}$ \\ ${ }^{1}$ Institute for Quantum Studies and Department of Physics, \\ Texas AËM University, College Station, TX, 77843 \\ ${ }^{2}$ Applied Physics and Materials Science Group, Eng. Quad., Princeton University, NJ, 08544
}

(Dated: July 4, 2021)

\begin{abstract}
We have shown that a coherently driven solid state medium can potentially produce strong controllable short pulses of $\mathrm{THz}$ radiation. The high efficiency of the technique is based on excitation of maximal $\mathrm{THz}$ coherence by applying resonant optical pulses to the medium. The excited coherence in the medium is connected to macroscopic polarization coupled to $\mathrm{THz}$ radiation. We have performed detailed simulations by solving the coupled density matrix and Maxwell equations. By using a simple $V$-type energy scheme for ruby, we have demonstrated that the energy of generated $\mathrm{THz}$ pulses ranges from hundreds of pico-Joules to nano-Joules at room temperature and micro-Joules at liquid helium temperature, with pulse durations from picoseconds to tens of nanoseconds. We have also suggested a coherent ruby source that lases on two optical wavelengths and simultaneously generates $\mathrm{THz}$ radiation. We discussed also possibilities of extension of the technique to different solid-state materials.
\end{abstract}

PACS numbers: Gy.42.50

\section{INTRODUCTION}

The search for efficient, high-power, inexpensive, compact, and room-temperature methods of generation of coherent teraherz $(\mathrm{THz})$ radiation is one of the main topics in modern optoelectronics and photonics [1]. Its importance is based on the fact that $\mathrm{THz}$ radiation has unique potential for a wide range of applications from diagnostics of different materials (including semiconductors, chemical compounds, biomolecules, and biotissues), imaging (for Imedical and security purposes), to remote atmospheric sensing and monitoring, astronomy, etc. 2, 3].

'In previous works we have focussed on a new approach to the problem of generation of short coherent $\mathrm{THz}$ pulsed - Tadiation by taking advantage of dramatic enhancement $>$ of the nonlinear response of a medium via maximal quantum coherence [4, 5, 6], created in atomic and molecular gàses with different level configurations (double- $\Lambda, \mathrm{V}-\Lambda$, Jlouble- $V$ schemes) by coherent laser radiaiton.

As is well-known, quantum coherence can dramatically change the optical properties of media. For example, lasing without population inversion (LWI) 7] and electromagnetically induced transparency (EIT) [8] have been demonstrated 9, 10 in coherently driven media. These unusual properties are employed for generation of electromagnetic radiation of different frequencies ranging from IR [1, 12] to UV [13], and gamma-rays [14]. Enhancement of coherent Raman scattering via maximal coherence has been demonstrated experimentally [15], and it has applications to enhanced real time spectroscopy (FAST CARS) 16]. Previously, the double $\Lambda$ scheme with near-maximal Raman coherence was used for highly efficient conversion of blue to ultraviolet light in $P b$ vapor 13]. A new type of gas-phase optical parametric oscillator 11] was suggested for frequency downconversion to generate $1.88 \mu \mathrm{m}$ radiation in $\mathrm{Pb}$ vapor, and efficient infrared upconversion [12] was suggested to convert infrared light with a $100 \mu \mathrm{m}$ wavelength to the visible. The first experimental demonstration supporting these ideas was performed in [4] where $5 \mu \mathrm{m}$ IR radiaiton was generated in $R b$ atomic vapor.

Many results in this area have been obtained in gases, but, recently, EIT has been generalized and extended to solids with a long-lived spin coherence 17], a class of solid materials, namely, rare-earth and transition metal ion doped dielectrics have been suggested as very attractive from the point of view of realization and applications of EIT. Several applications of EIT to improve performance of solid state lasers have been already proposed 18, 19]. LWI, first realized in a gas medium [9], recently has been demonstrated in solids as well 20].

In this paper we extend the approach of generation of $\mathrm{THz}$ radiation via resonantly induced coherence to solid state media. The coherence at the corresponding transition can be induced by coherent optical fields as shown in Fig. 10 Electronic levels of the THz transition are coupled by a pair of optical fields with Rabi frequencies $\Omega_{1}$ and $\Omega_{2}$ to a common ground state forming a $\mathrm{V}$ system of energy 
levels. Coherence $\rho_{c b}$ induced by the optical fields at the $\mathrm{THz}$ transition gives rise to polarization provided that the corresponding dipole moment is not zero, which will radiate out a $\mathrm{THz}$ pulse with Rabi frequency $\Omega_{3}$. This method differs from the one proposed in [5, 6] for gaseous media where the optically prepared $\mathrm{THz}$ coherence produces no macroscopic polarization of gaseous media at a $\mathrm{THz}$ transition (this is the manifestation of symmetry with respect to inversion), the reason is that an electrically allowed dipole moment at the two-photon transition is zero since it is typically forbidden (although, it is important to note here that the magnetic dipole moment can be non-zero even in gases, we discuss this opportunity later). For doped solids having sites with no inversion symmetry is common, such as the one occupied by $\mathrm{Cr}^{3+}$ (simplified energy level structure is shown in Fig.(11)) in ruby, the corresponding two-photon transition dipole moment is not zero, resulting in all three transitions in the $V$ scheme being allowed. It is worth mentioning that using nonlinear optical mixing in a semiconductor heterostructure for generation of few-cycle $\mathrm{THz}$ pulses was proposed in [23].

In ruby closely spaced $R_{1}$ and $R_{2}$ lines arise from transitions between the ground state of the $\mathrm{Cr}^{3+}$ ion $\left({ }^{4} \mathrm{~A}_{2}\right)$ and its first excited state $\left({ }^{2} \mathrm{E}\right)$. Each of these cubic-field states is split by the trigonal crystal field and spin-orbit coupling into a pair of Kramers doublets, $0.38 \mathrm{~cm}^{-1}$ apart in the ground state and $29.14 \mathrm{~cm}^{-1}$ apart in the ${ }^{2} \mathrm{E}$ state. The width of the $\mathrm{R}$ lines is about $11 \mathrm{~cm}^{-1}$ at room temperature and $0.15 \mathrm{~cm}^{-1}$ at the temperature of liquid nitrogen. The transitions from the ${ }^{4} \mathrm{~A}_{2}$ ground state to levels ${ }^{2} \mathrm{E}$ are spin-forbidden, and have oscillator strength $\sim 7.5 \cdot 10^{-7}\left(\mathrm{R}_{1}, \mathrm{R}_{2}\right.$ line peak cross-section is $\sim(1.2-1.4) \cdot 10^{-18} \mathrm{~cm}^{2}$ at the temperature of liquid nitrogen and $\sim 4 \cdot 10^{-20} \mathrm{~cm}^{2}$ at room temperature). They are predominantly electric-dipole in nature, since the inversion symmetry of a cubic crystal field is broken by crystal field trigonal distortions and odd-parity lattice vibrations. Namely, the odd-parity component of the crystal field at the $\mathrm{Cr}^{3+}$ ion mixes odd-parity states of high energy into the even parity d states between which R-transitions are observed and makes them weakly allowed. The magnetic-dipole contribution to the R-lines is estimated to be $\sim 1 / 30$ of the strength of the elecricdipole transitions [26].

The $29 \mathrm{~cm}^{-1}$ transition in ruby has history which is as rich as the ruby laser itself. It was considered promising for realization of a quantum counter for farinfrared radiation (FIR) 27] on one hand, and as a system very convenient to study interaction processes of non-equilibrium phonons with two-level electronic systems (so-called phonon spectrometer), on the other 28]. There was also a proposal to make a far-infrared laser at this transition pumping via the $\mathrm{R}_{2}$ line, but due to the unfavorable ratio of relaxation rates (fast relaxation at the FIR and slow at the optical $R_{1}$ transition), the laser could operate only at liquid helium temperature and the estimated gain did not exceed losses 29].

It is interesting that $29 \mathrm{~cm}^{-1}$ FIR was produced by non-linear mixing of two ruby laser beams, one emitting at $\mathrm{R}_{1}$ and another at $\mathrm{R}_{2}$, in $\mathrm{LiNbO}_{3}$ 30 and in $\mathrm{ZnTe}$ crystals [31], with the efficiency $\eta \sim 10^{-9}$. The method proposed in the present work would allow pulsed $\mathrm{THz}$ radiation to be easily produced from a dual-color ruby laser itself, operating at both $\mathrm{R}_{1}$ and $\mathrm{R}_{2}$ lines, thus significantly miniaturizing the system.

The outline of the paper is the following: in the next section we describe a theoretical model used to calculate parameters of generated $\mathrm{THz}$ pulses. In section III we consider possibilities of phase-matching for fields in different regimes (CW and short pulses). In section IV we make estimates showing the perspective of the suggested scheme as well as discuss a proposed experiment with a standard femto-second laser setup. Next we show that the system we have considered is not limited, i.e. there are many systems to which our theory can be applied. Finally we compare the efficiency of our scheme with the methods of generation of $\mathrm{THz}$ radiation used nowadays and possible applications that are opened due to the high efficiency of the proposed technique.

\section{THEORETICAL MODEL}

\section{A. Real system}

The ruby system shown in Fig.(1) includes eight states, four in the ground ${ }^{4} A_{2}$ and four in the excited ${ }^{2} E$ one. In our theoretical analysis we describe it using only three levels as in Fig.(10), since this simple model takes into account all essential physics and allows us to make estimates of expected $\mathrm{THz}$ radiation characteristics and required optical field parameters.

For the proposed technique to work we need to make sure that an efficient $V$ scheme can be realized. In the case of a single fs pulse, driving both optical transitions simultaneously, we will have only one polarization, for example, linear. For two pulses we can choose each pulse polarization separately. Selection rules for the right circular and left circular polarizations are shown in Fig.(2) 
24]. As one can see, neither for the right, nor for the left circular polarization a $V$ scheme involving $\mathrm{R}_{1}$ and $\mathrm{R}_{2}$ transitions can be organized. For a linear polarization perpendicular to the optic axis, which is an equal sum or difference of the right and left ones, there are four $V$ schemes possible, shown in Fig.(2b).

Taking into account the relation between the coefficients $\mathrm{C}^{+}$and $\mathrm{C}^{-}: C^{+} C^{-*}=-C^{-} C^{+*}$, one can see that the schemes $\mathrm{A}$ and $\mathrm{C}$ cancel each other, since the products of the matrix elements are opposite $\left( \pm 4 C^{+} C^{-*} / 9\right.$ for $\mathrm{A}$ and $\mathrm{C}$, respectively). On the other hand, for schemes $\mathrm{B}$ and $\mathrm{D}$ the products are the same $\left(-2 \sqrt{2} C^{+} C^{-*} / 9\right)$. These two $V$ schemes will interfere constructively and induce the $\mathrm{THz}$ coherence.

\section{B. Simplified model}

The interaction Hamiltonian for the system shown in Fig. (10) is given by

$$
\begin{gathered}
V_{I}=-\hbar\left[\Omega_{1} e^{-i \omega_{b a} t}|b><a|+\Omega_{2} e^{-i \omega_{c a} t}|c><a|+h . c .\right] \\
-\hbar\left[\Omega_{3} e^{-i \omega_{c b} t}|c><b|+h . c .\right]
\end{gathered}
$$

where $\Omega_{i}=p_{\mu \nu} \mathcal{E}_{i} / \hbar$ is the Rabi frequency of the respective field, $\mu \nu=b a, c a, c b$ and $i=1,2,3 ; p_{b a}$ and $p_{c a}$ are the electric dipole matrix elements of the optical transitions $b \leftrightarrow a$ and $c \leftrightarrow a$, and $p_{c b}$ of the $\mathrm{THz}$ transition $c \leftrightarrow b$, respectively; $\omega_{b a}, \omega_{c a}$ and $\omega_{c b}$ are the frequencies of the electronic and $\mathrm{THz}$ transitions; $\mathcal{E}_{i}$ is the amplitude of the respective electromagnetic field.

The time-dependent density matrix equations are

$$
\frac{\partial \rho}{\partial t}=-\frac{i}{\hbar}[H, \rho]-\frac{1}{2}(\Gamma \rho+\rho \Gamma),
$$

where $\Gamma$ is the relaxation matrix, and $H$ is the total Hamiltonian.

To estimate the efficiency of $\mathrm{THz}$ radiation generation and required laser field intensities we first consider the system (2) in the limit of ultrashort pulses, which are short compared to relaxation times of the system, and neglect propagation effects for the optical fields. In this simplified picture we assume that the system is driven by two resonant fields with equal time-dependent Rabifrequencies $\Omega_{1}=\Omega_{2}=\Omega(t)$ (assumed real). In the case of a V-scheme considered in this work the STIRAP technique, proposed in 5, 6] for maximal molecular coherence excitation in a $\Lambda$ energy system, is not applicable. In the case of the $\mathrm{V}$ scheme maximal coherence is excited by two fields of equal Rabi frequency and duration, acting simultaneouly. The system is then described by the following set of equations

$$
\begin{gathered}
\frac{d \sigma_{c a}}{d t}=i \Omega\left(\rho_{a}-\rho_{c}\right)-i \Omega \sigma_{c b}, \\
\frac{d \sigma_{b a}}{d t}=i \Omega\left(\rho_{a}-\rho_{b}\right)-i \Omega \sigma_{c b}^{*}, \\
\frac{d \sigma_{c b}}{d t}=i \Omega\left(\sigma_{b a}^{*}-\sigma_{c a}\right),
\end{gathered}
$$

$$
\frac{d \rho_{a}}{d t}=i \Omega\left(\sigma_{b a}+\sigma_{c a}\right)-i \Omega\left(\sigma_{b a}^{*}+\sigma_{c a}^{*}\right),
$$

$$
\begin{gathered}
\frac{d \rho_{b}}{d t}=i \Omega\left(\sigma_{b a}^{*}-\sigma_{b a}\right), \\
\rho_{c}=1-\rho_{a}-\rho_{b} .
\end{gathered}
$$

Due to the symmetry of the model system $\rho_{b}=\rho_{c}, \sigma_{b a}=$ $\sigma_{c a}$ and $\sigma_{c b}$ - real. Let us introduce new variables

$$
\begin{gathered}
\alpha=\left(\sigma_{b a}+\sigma_{c a}\right) / 2=\sigma_{b a}, \\
\beta=\left(\sigma_{c b}+\sigma_{c b}^{*}\right) / 2=\operatorname{Re}\left(\sigma_{c b}\right)=\sigma_{c b},
\end{gathered}
$$

for which, taking into account (5) and (6), the following relation can be obtained

$$
\frac{d}{d t}\left(\rho_{a}+2 \beta\right)=0
$$

giving $\rho_{a}+2 \beta=1$. For the new variables we arrive at the following system of equations

$$
\begin{gathered}
\frac{d \alpha}{d t}=i \Omega-4 i \Omega \beta, \\
\frac{d \beta}{d t}=2 i \Omega\left(\alpha^{*}-\alpha\right),
\end{gathered}
$$

which can be further simplified if we substitute $\xi=\alpha-\alpha^{*}$

$$
\begin{gathered}
\frac{d \xi}{d t}=2 i \Omega-8 i \Omega \beta \\
\frac{d \beta}{d t}=-2 i \Omega \xi
\end{gathered}
$$


The solution of this system is

$$
\begin{gathered}
\xi=\frac{\sqrt{2} i}{2} \sin \left(2 \sqrt{2} \int_{-\infty}^{t} \Omega\left(t^{\prime}\right) d t^{\prime}\right), \\
\beta=\sigma_{c b}=\frac{1}{4}\left(1-\cos \left(2 \sqrt{2} \int_{-\infty}^{t} \Omega\left(t^{\prime}\right) d t^{\prime}\right)\right) .
\end{gathered}
$$

It follows from Eq.(17) that maximal coherence $\sigma_{c b}=$ 0.5 will be excited by a pair of pulses with area $S=$ $2 \sqrt{2} \int_{-\infty}^{\infty} \Omega(t) d t=\pi$ each. Taking pulses of a Gaussian shape $\Omega=\Omega_{0} \exp \left(-t^{2} / 2 \tau^{2}\right)$ with the area of the pulse $S=4 \sqrt{\pi} \Omega_{0} \tau$, we will have the maximal coherence excited when $\Omega_{0} \tau=\sqrt{\pi} / 4 \approx 0.443$. The excited coherence will give rise to the polarization which will radiate out a coherent $\mathrm{THz}$ pulse. It is worth noting that this mechanism is similar to free-induction decay (FID) 25] in photon echo: excitation of a maximal coherence in a two-level system by a $\pi / 2$ pulse, followed by emission of a coherent pulse. The difference is that typically in solids the FID decay is governed by the dephasing due to inhomogeneous broadening of the transition leading to decay of the corresponding coherence during the time $\sim W_{i n h}^{-1}$. In the case of ruby the $\mathrm{THz}$ transition is homogeneously broadened even at liquid helium temperature, so the decay of the $\mathrm{THz}$ coherence is determined by the homogeneous width of the corresponding transition.

For two long pulses, which can be considered as CW (in the sense that optical and $\mathrm{THz}$ coherences approach the steady-state), with approximately equal Rabi frequencies $\Omega_{1} \approx \Omega_{2}$, resonant with the corresponding optical transitions, we get the $\mathrm{THz}$ coherence

$$
\sigma_{c b}=\frac{2 \Omega_{2} \Omega_{1}^{*} \exp \left(-\frac{6\left|\Omega_{1}\right|^{2} t}{\gamma+\left(\left|\Omega_{1}\right|^{2}+\left|\Omega_{2}\right|^{2}\right) / \gamma_{c b}}\right)}{\gamma_{c b}\left(\gamma+\left(\left|\Omega_{1}\right|^{2}+\left|\Omega_{2}\right|^{2}\right) / \gamma_{c b}\right)},
$$

where $\gamma_{b a}=\gamma_{c a}=\gamma$ and $\gamma_{c b}$ are the optical and $\mathrm{THz}$ coherence decay rates, respectively. In this case the maximal $\mathrm{THz}$ coherence $\left|\sigma_{c b}\right|=1$ will be excited provided that $\left(\left|\Omega_{1}\right|^{2}+\left|\Omega_{2}\right|^{2}\right) \gg \gamma \gamma_{c b}$, which is a usual steady-state EIT threshold condition.

Let us now estimate the peak intensity and energy of the optical pulses necessary to excite required coherence. The peak intensity of the pulse is expressed in terms of the peak Rabi-frequency as

$$
I_{\text {peak }}=\frac{2 \pi \hbar c \Omega_{0}^{2}}{\gamma n \lambda \sigma_{a b s}},
$$

where $\gamma$ is the width of an optical transition, $n$ is the refractive index at the optical wavelength, $\lambda$ is the optical wavelength, and $\sigma_{a b s}$ is the corresponding absorption cross-section. The energy of a single pulse can be calculated as

$$
E_{o p t} \approx \frac{2 \pi \sqrt{\pi} \hbar c A_{o p t}}{\gamma n \lambda \sigma_{a b s}} \Omega_{0}^{2} \tau,
$$

where $A_{\text {opt }}$ is the laser beam cross-section.

\section{PROPAGATION: PHASE-MATCHING GEOMETRY FOR FIELDS}

A complete self-consistent calculation also includes field propagation equations

$$
\frac{\partial \Omega_{\alpha}}{\partial z}+\frac{n_{\alpha}}{c} \frac{\partial \Omega_{\alpha}}{\partial t}=-\kappa_{\alpha} \Omega_{\alpha}+i \eta_{\alpha} \rho_{\alpha},
$$

where index $\alpha=1,2,3$ indicates all fields and corresponding polarizations, $\eta_{\alpha}=2 \pi \omega_{\alpha} N \mu_{\alpha}^{2} / n_{\alpha} c \hbar$ is the corresponding coupling constant, $\omega_{\alpha}$ are the frequencies of the optical and $\mathrm{THz}$ fields, $N$ is the density of the medium, $c$ is the speed of light in vacuum, $n_{\alpha}$ is the corresponding refractive index, and $\kappa_{\alpha}$ are losses for the field during propagation in the crystal due to scattering, diffraction, or non-resonant absorption. For optical fields these losses are usually small, but for the $\mathrm{THz}$ field in free space the diffraction losses given by $\kappa_{3}=\lambda_{3} / D^{2}$ should be taken into account. To avoid diffraction losses the crystal can be placed in a waveguide for $\mathrm{THz}$ radiation, then the distribution of the field mode should be taken into account.

Propagation effects are important for nonlinear interactions such as wave mixing. Let us consider two regimes: CW, when the spectral widths of pulses is smaller than the splitting between the levels of the $\mathrm{THz}$ transition, and pulsed one, when the spectral width of pulses exceeds the splitting between the levels.

In the CW regime the field $\Omega_{3}$ at the output of the crystal is given by (assuming that the $\mathrm{THz}$ pulse propagates in $z$ direction)

$$
\begin{gathered}
\Omega_{3}=i e^{-\kappa_{3} L} \int_{0}^{L} \eta \sigma_{c b} e^{\left(i \delta k+\kappa_{3}\right) z} d z=i \eta_{3} \sigma_{c b} \frac{e^{i \delta k L}-e^{-\kappa_{3} L}}{i \delta k+\kappa_{3}}= \\
=i \eta_{3} \sigma_{c b} L e^{i \delta k L / 2} \frac{\sin (\delta k L / 2)}{(\delta k L / 2)},
\end{gathered}
$$

if losses are neglected. Here $L$ is the length of the crystal, and the prefactor describes phase-matching $\left(\delta k=k_{2 z}-\right.$ $\left.k_{1 z}-k_{3}\right)$. The direction of $\mathrm{THz}$ propagation is given by the phase-matching condition (see Fig.3)

$$
\overrightarrow{k_{2}}-\overrightarrow{k_{1}}=\overrightarrow{k_{3}}
$$


To satisfy the phase-matching condition $\delta k=0$ for the THz pulse to propagate in $z$ direction (along the crystal) the angle between the optical fields has to be adjusted according to the equantion

$$
k_{1}^{2}+k_{2}^{2}-2 k_{1} k_{2} \cos \phi=k_{3}^{2}, \cos \phi=\frac{k_{1}^{2}+k_{2}^{2}-k_{3}^{2}}{2 k_{1} k_{2}} .
$$

Rewriting a condition for optical and $\mathrm{THz}$ frequencies $\omega_{2}-\omega_{1}=\omega_{3}$ in the form of $k_{3}=\left(k_{2}-k_{1}\right) n_{3} / n_{1}$, we obtain

$$
\phi=\sqrt{\frac{n_{3}^{2}-n_{1}^{2}}{n_{1}^{2}}} \frac{\lambda_{1}}{\lambda_{3}},
$$

where $\lambda_{1(3)}$ is the wavelength of the optical $(\mathrm{THz})$ field. For the case of ruby the refractive index for $\mathrm{THz}$ radiation is twice larger than the one for optics, $n_{3} \approx 2 n_{1,2}$ 29], then the angle between directions of propagation for optical fields $\phi=3 \cdot 10^{-3}$. The angle between the $k_{1(2)}$ vectors and the $\mathrm{THz}$ field propagation direction is $\pi / 3$ in

this case. This provides an interesting opportunity to design a dual-wavelength ruby laser working simultaneously at $R_{1}$ and $R_{2}$ lines that can also generate $\mathrm{THz}$ radiation, as is shown in Fig 3 . The crystal will simultaneously serve as a $\mathrm{THz}$ waveguide in this case.

Now we turn to a short pulse excitation regime. For many femtosecond laser experimental setups it would be an interesting extension to include an option of $\mathrm{THz}$ generation. The phase-matching in this case can be obtained by excitation of $\mathrm{THz}$ coherence with two optical beams with the same frequencies but different directions of propagation. It is instructive to show how it works in this case. Let us consider the problem in the simplest case of lowest order of perturbation in coherent pumping fields. The density matrix equations look like

$$
\begin{array}{r}
\sigma_{a b}^{\cdot}=-i \Omega_{1} e^{-i k_{x} x+i k_{z} z}-i \Omega_{2} e^{-i k_{x} x-i k_{z} z}, \\
\sigma_{c a}^{\cdot}=i \Omega_{1} e^{i k_{x} x-i k_{z} z}+i \Omega_{2} e^{i k_{x} x-i k_{z} z},
\end{array}
$$

$$
\sigma_{c b}^{\cdot}=i \sigma_{a b}\left(\Omega_{1}(t) e^{i k_{x} x-i k_{z} z}+\Omega_{2}(t) e^{i k_{x} x+i k_{z} z}\right)-i \sigma_{c a}\left(\Omega_{1}(t) e^{-i k_{x} x+i k_{z} z}+\Omega_{2}(t) e^{-i k_{x} x-i k_{z} z}\right) .
$$

A solution of this set of equations is given by

$$
\sigma_{c b}=2 \int_{-\infty}^{t} d t^{\prime} \int_{-\infty}^{t^{\prime}} d t^{\prime \prime}\left(\Omega_{1}\left(t^{\prime}\right) \Omega_{1}\left(t^{\prime \prime}\right)+\Omega_{2}\left(t^{\prime}\right) \Omega_{2}\left(t^{\prime \prime}\right)+\Omega_{1}\left(t^{\prime}\right) \Omega_{2}\left(t^{\prime \prime}\right) \cos 2 k_{z} z+\Omega_{1}\left(t^{\prime \prime}\right) \Omega_{2}\left(t^{\prime}\right) \cos 2 k_{z} z\right)
$$

One can see that the phase-matching between the optical fields and the $\mathrm{THz}$ field is achieved by appropriate tilting of the optical beams at the angle $\phi \approx 2 k_{z} / k_{x}=k_{3} / k_{1}=$ $\lambda_{1} n_{3} / \lambda_{3} n_{1}$; in particularly, for ruby $\phi \approx 10^{-3}$.

\section{ESTIMATION OF ENERGY OF GENERATED THZ FIELD}

First we analize the optimal conditions at which the maximal $\mathrm{THz}$ coherence can be induced in ruby by the optical fields at both room and liquid helium tempera- tures. For room temperature ruby $\gamma=\gamma_{c b}=5.5 \mathrm{~cm}^{-1}$ 32], $n=1.76, \lambda=694.3 \mathrm{~nm}$, and $\sigma_{a b s}=4 \cdot 10^{-20} \mathrm{~cm}^{2}$ 26]. In the case of ultrashort driving pulses (100 fs-1 ps) the shortest $\mathrm{THz}$ pulse that can be generated in this system has a duration $\sim \gamma_{c b}^{-1} \sim 1$ ps. With the optical pulses of the same duration $\tau=1$ ps the Rabi frequency $\Omega_{0}=4.43 \cdot 10^{11} \mathrm{~s}^{-1}$ is required to produce the maximal coherence, corresponding to $I_{\text {peak }} \sim 10^{12} \mathrm{~W} / \mathrm{cm}^{2}$, which is still below a damage threshold of ruby for such duration of the pulses [33], but we make a conservative estimate for lower intensities $I_{\text {peak }} \sim 100 \mathrm{GW} / \mathrm{cm}^{2}$, certainly below 
the threshold, corresponding to the peak Rabi frequency $\Omega_{0}=2 \cdot 10^{11} \mathrm{~s}^{-1}$. For long driving pulses (10 ps $\left.-1 \mathrm{~ns}\right)$ to excite the maximal coherence $\left|\sigma_{c b}\right|=1$ would require $\Omega_{1,2}^{2} \gg \gamma \gamma_{c b}$ resulting in the Rabi frequency $\Omega_{1,2} \sim 10^{12}$ $\mathrm{s}^{-1}$, corresponding to intensities exceeding the ruby damage threshold for pulses of such duration. For long pulses (1-10 ns) the damage threshold intensity is in the range $I_{\text {peak }} \sim 20-30 \mathrm{GW} / \mathrm{cm}^{2}$ [34], which corresponds to the Rabi frequency $\Omega_{1,2} \sim 10^{11} \mathrm{~s}^{-1}$. This value is used in the estimates below.

At low temperatures $(1.8-4.2 \mathrm{~K})$ the $\mathrm{THz}$ coherence lifetime is significantly larger, $\gamma_{c b}^{-1} \sim 500$ ps [35], which means that with ultrashort pumping pulses (in this case of 10 ps-1 ns duration), the maximal coherence will be excited with only $\Omega_{0} \sim 10^{10} \mathrm{~s}^{-1}$, requiring $I_{\text {peak }} \sim 100$ $\mathrm{MW} / \mathrm{cm}^{2}$ pulse intensity. For long pulses (1-10 ns) the maximal coherence will be excited when $\Omega_{1,2}^{2} \sim \gamma \gamma_{c b}$, and since at low temperatures $\gamma \sim 0.05 \mathrm{~cm}^{-1}$ [36], it results in $\Omega_{1,2} \sim 5 \cdot 10^{9} \mathrm{~s}^{-1}$, requiring $I_{\text {peak }} \sim 30 \mathrm{MW} / \mathrm{cm}^{2}$.

The next question is the energy of the generated $\mathrm{THz}$ pulse, which can be estimated from the propagation equation

$$
\frac{\partial \Omega_{3}}{\partial z}+\frac{n_{3}}{c} \frac{\partial \Omega_{3}}{\partial t}=\frac{2 \pi \omega_{3} i N \mu_{c b}^{2}}{n_{3} c \hbar} \sigma_{c b}-\kappa \Omega_{3},
$$

where $N$ is the density of $\mathrm{Cr}^{3+}$ ions, $\hbar \omega_{3}=29$ $\mathrm{cm}^{-1}(0.87 \mathrm{THz})$, the dipole moment of the farinfrared transition can be calculated using measured experimentally low-temperature (LT) parameters $\mu_{c b}^{2}=$ $\gamma_{c b}^{L T} c \hbar n_{3} \sigma_{a b s, T H z}^{L T} / 2 \pi \omega_{3}$, and $\kappa$ is the non-resonant absorption coefficient of the sapphire host at the $\mathrm{THz}$ wavelength.

Assuming that the crystal sample is side-pumped ho- mogeneously, so that the $\mathrm{THz}$ coherence $\sigma_{c b}$ does not depend on the propagation coordinate $z$, and phasematching conditions are satisfied we arrive at the equation

$$
\frac{\partial \Omega_{3}}{\partial z}+\frac{n_{3}}{c} \frac{\partial \Omega_{3}}{\partial t}=i \eta \sigma_{c b}(t)-\kappa \Omega_{3},
$$

where $\eta=N \gamma_{c b}^{L T} \sigma_{a b s, T H z}^{L T}$, so that at the end of the crystal the Rabi-frequency of the $\mathrm{THz}$ pulse is

$$
\Omega_{3}(t, L)=i \eta \int_{z_{0}}^{L} \sigma_{c b}\left(t-\frac{z-z^{\prime}}{c} n_{3}\right) e^{-\kappa\left(z-z^{\prime}\right)} d z^{\prime}
$$

where $z_{0}=\max \left(0, L-t c / n_{3}\right)$. For the ultrashort pumping pulses we can model $\sigma_{c b}(t)=\sigma_{c b}^{\max } \exp \left(-\gamma_{c b} t\right)$ at $t>0$ and $\sigma_{c b}=0$ at $t<0$ for an estimate. Eq.(32) gives

$$
\Omega_{3}=i \eta \sigma_{c b}^{\max } \frac{e^{-\gamma_{c b} t}}{\gamma_{c b} n_{3} / c-\kappa}\left(e^{\left(\gamma_{c b} n_{3} / c-\kappa\right) c t / n_{3}}-1\right)
$$

for $t<L n_{3} / c$ and

$$
\Omega_{3}=i \eta \sigma_{c b}^{\max } \frac{e^{-\gamma_{c b} t}}{\gamma_{c b} n_{3} / c-\kappa}\left(e^{\left(\gamma_{c b} n_{3} / c-\kappa\right) L}-1\right)
$$

for $t>L n_{3} / c$.

Eqs. (33)- (34) then allow one to calculate the energy of the $\mathrm{THz}$ pulse

$$
E_{T H z}=\frac{\hbar \omega_{3} A}{\gamma_{c b}^{L T} n_{3} \sigma_{a b s, T H z}^{L T}} \int_{0}^{\infty}\left|\Omega_{3}\right|^{2} d t=
$$

$$
=\frac{\hbar \omega_{3} A N^{2} \gamma_{c b}^{L T} \sigma_{a b s, T H z}^{L T}}{n_{3}}\left(\sigma_{c b}^{\max }\right)^{2}\left(\frac{1}{2 \kappa \gamma_{c b}\left(\kappa+\gamma_{c b} n_{3} / c\right)}-\frac{e^{-2 \kappa L}}{2 \kappa \gamma_{c b}\left(\gamma_{c b} n_{3} / c-\kappa\right)}+\frac{e^{-\left(\kappa+\gamma_{c b} n_{3} / c\right) L}}{\gamma_{c b}\left(\left(\gamma_{c b} n_{3} / c\right)^{2}-\kappa^{2}\right)}\right),
$$

where $A$ is the crystal cross-section along THz pulse propagation direction. For two pulses of 1 ps duration with $I_{\text {peak }} \sim 100 \mathrm{GW} / \mathrm{cm}^{2}$ the maximal induced coherence is $\sigma_{c b}^{\max } \approx 0.21$. Taking $N=1.6 \cdot 10^{19} \mathrm{~cm}^{-3}$ for $0.05 \%$ doped ruby, and known from low-temperature measurements $\gamma_{c b}^{L T}=2 \cdot 10^{9} \mathrm{~s}^{-1}, \sigma_{a b s, T H z}^{L T}=3 \cdot 10^{-19} \mathrm{~cm}^{2}[35]$, and room temperature parameters $\gamma_{c b}=10^{12} \mathrm{~s}^{-1}$ and $\kappa=0.4-0.5 \mathrm{~cm}^{-1}$ [29], $n_{3}=3.5$ [29], and considering the crystal of $1 \mathrm{~cm} \times 1 \mathrm{~cm} \times 0.1 \mathrm{~cm}$ size with the $A=1 \mathrm{~cm} \times$ $0.1 \mathrm{~cm} \mathrm{THz}$ emitting cross-section and $L=1 \mathrm{~cm}$ length, we arrive at $E_{T H z} \approx 630$ pJ. The energy can be higher for higher optical peak intensities, approaching several nJ for $I_{\text {peak }} \sim 10^{12} \mathrm{~W} / \mathrm{cm}^{2}$. For low temperatures $(1.8-4.2 \mathrm{~K})$ the parameters are: $\gamma_{c b}=2 \cdot 10^{9} \mathrm{~s}^{-1}, \sigma_{a b s, \text { THz }}=3 \cdot 10^{-19}$ 
$\mathrm{cm}^{2}, \kappa=0.01 \mathrm{~cm}^{-1}$ [29], and the maximal coherence $\sigma_{c b}=0.5$ can be easily excited. Due to stronger absorption at low temperatures $\left(\sigma_{a b s} \sim 10^{-18} \mathrm{~cm}^{2}[26]\right)$, half of the optical intensity will be absorbed at $\approx 0.5 \mathrm{~mm}$ for $0.05 \% \mathrm{Cr}^{3+}$ density, therefore, for this estimate we take a crystal with dimensions $1 \mathrm{~cm} \times 0.1 \mathrm{~cm} \times 0.05 \mathrm{~cm}$ size with the THz emitting cross-section $A=0.1 \mathrm{~cm} \times 0.05 \mathrm{~cm}$ and $L=1 \mathrm{~cm}$ length which results in $E_{T H z} \sim 7.5 \mu \mathrm{J}$.

Let us also make an estimate for fs pulse duration. Since powerful fs Ti:Sapphire lasers are readily available in many laboratories nowadays this is of interest. In this case the coherence can be excited by just one pulse of 100 fs duration, since its spectral width is larger than the $\mathrm{THz}$ transition splitting. Although, to satisfy phase-matching conditions, it is necessary to split it in two beams hiting the crystal at slightly different angles. Keeping the same peak intensity $I_{\text {peak }} \approx 100 \mathrm{GW} / \mathrm{cm}^{2}$ we obtain a smaller pulse area $S=0.14$, giving the coherence $\sigma_{c b}^{\max } \sim 2.5$. $10^{-3}$ and resulting in $\mathrm{THz}$ pulse energy of $\approx 100 \mathrm{fJ}$ at room temperature.

For the CW pumping case we have, in analogy with the short pulse pumping,

$$
E_{T H z}=\frac{\hbar \omega_{3} A N^{2} \gamma_{c b}^{L T} \sigma_{a b s, T H z}^{L T}}{n_{3}}\left(\sigma_{c b}^{\max }\right)^{2}\left(\frac{1}{2 \kappa G\left(\kappa+G n_{3} / c\right)}-\frac{e^{-2 \kappa L}}{2 \kappa G\left(G n_{3} / c-\kappa\right)}+\frac{e^{-\left(\kappa+G n_{3} / c\right) L}}{G\left(\left(G n_{3} / c\right)^{2}-\kappa^{2}\right)}\right)
$$

where from Eq. (18)
$2 \Omega_{2}^{*} \Omega_{1} /\left(\gamma \gamma_{c b}+\left|\Omega_{1}\right|^{2}+\left|\Omega_{2}\right|^{2}\right)$
$6\left|\Omega_{1}\right|^{2} /\left(\gamma+\left(\left|\Omega_{1}\right|^{2}+\left|\Omega_{2}\right|^{2}\right) / \gamma_{c b}\right)$. and $\quad$ In the limit $\Omega_{1,2}^{2} \geq \gamma \gamma_{c b}$ the maximal coherence is excited $\sigma_{c b}=1$, which would require, though, experimentally unfeasible Rabi frequency $\Omega_{1,2} \sim 10^{12} \mathrm{~s}^{-1}$ at room temperature, resulting in $I_{\text {peak }} \sim 10^{12} \mathrm{~W} / \mathrm{cm}^{2}$. For such long pulses (10 ps $-1 \mathrm{~ns})$ the damage threshold intensity for ruby is $I^{\text {peak }} \sim 20-30 \mathrm{GW} / \mathrm{cm}^{2}$, which corresponds to the Rabi frequency $\Omega_{1,2} \sim 10^{11} \mathrm{~s}^{-1}$. For this Rabi frequency and room temperature decay rates $\gamma \approx \gamma_{c b}=10^{12} \mathrm{~s}^{-1}$, the ratio $\Omega_{1,2}^{2} / \gamma \gamma_{c b} \sim 10^{-2}$ and the resulting $\mathrm{THz}$ coherence $\left|\sigma_{c b}\right| \sim 10^{-2}$ for a $10 \mathrm{ps}$ pulse. The THz pulse energy will be $E_{T H z} \sim 300 \mathrm{pJ}$. At low temperatures the maximal coherence $\sigma_{c b}=1$ can be excited by long pulses (1-10 ns), which would give energy of a THz pulse $E_{T H z} \sim 8$ $\mu \mathrm{J}$.

Finally we estimate the total energy of optical pulses using Eq.(20) and overall conversion efficiency to $\mathrm{THz}$ radiation. For room temperature 1 ps pulses pumping the side of the crystal with $A_{o p t}=1 \mathrm{~cm} \times 0.1 \mathrm{~cm}$ size, the energy in one pulse according to Eq.(20) is $E_{\text {opt }} \approx$ $27 \mathrm{~mJ}$, for two pulses, $54 \mathrm{~mJ}$, respectively. We have to take into account that only $\left(1-\exp \left(-\sigma_{a b s} N L_{o p t}\right)\right)$ of the incident energy is absorbed, where $L_{\text {opt }}$ is the size of the crystal along which the optical pulses propagate. For room temperature $\sigma_{a b s}=4 \cdot 10^{-20} \mathrm{~cm}^{2}, N=1.6 \cdot 10^{19}$ $\mathrm{cm}^{-3}$ and $L_{o p t}=1 \mathrm{~cm}$, the absorbed fraction is 0.47 , so about twice the energy calculated above is required, resulting in $\sim 110 \mathrm{~mJ}$ total energy. For a pair of 100 fs pulses the same reasoning leads to the total energy of $\sim 11 \mathrm{~mJ}(2.7 \mathrm{~mJ}$ in one pulse from Eq.(20) $)$. For long pulses the total required energy is $300 \mathrm{~mJ}$.

The expected values of the energy of $\mathrm{THz}$ pulses, the peak amplitude of the THz field if focused to a spot of 300 $\mu \mathrm{m}$ size, required pumping radiation energy and duration and efficiency of optical to $\mathrm{THz}$ energy conversion for room temperature ruby are summurized in Table $\square$

At low temperatures as was already discussed above $55 \%$ of optical intensity is absorbed at $L_{\text {opt }}=0.05 \mathrm{~cm}$. Taking $A_{o p t}=1 \mathrm{~cm} \times 0.1 \mathrm{~cm}$ we obtain from Eq. (20) for short pulses $E_{\text {opt }} \approx 13.5 \mathrm{~mJ}$ in one pulse, giving total required energy of $54 \mathrm{~mJ}$. For long pulses the estimate gives $E_{\text {opt }}=67.5 \mathrm{~mJ}$ in one pulse and $270 \mathrm{~mJ}$ total energy for long pulses.

The parameters of $\mathrm{THz}$ pulses: energy, peak amplitude if focused to a spot of $300 \mu \mathrm{m}$ size, and required optical pulses energy and duration at low temperatures are summurized in Table II. 
TABLE I: Estimates of the THz pulse energy, peak THz field amplitude, required optical pulse parameters and THz radiation generation efficiency in ruby at room temperature

\begin{tabular}{ccc}
\hline \hline ultrashort & pulses $(100 \mathrm{fs}-1 \mathrm{ps})$ & long pulses (10 ps-100 ps) \\
\hline $100 \mathrm{fs}$ & $1 \mathrm{ps}$ & $10 \mathrm{ps}$ \\
\hline$\sigma_{c b} \sim 2.5 \cdot 10^{-3}$ & $\sigma_{c b}=0.21$ & $\sigma_{c b} \sim 10^{-2}$ \\
$E_{T H z} \sim 100 \mathrm{fJ}$ & $E_{T H z} \sim 630 \mathrm{pJ}$ & $E_{T H z} \sim 300 \mathrm{pJ}$ \\
$\mathcal{E}_{T H z} \sim 300 \mathrm{~V} / \mathrm{cm}$ & $\mathcal{E}_{T H z} \sim 23 \mathrm{kV} / \mathrm{cm}$ & $\mathcal{E}_{T H z} \sim 5 \mathrm{kV} / \mathrm{cm}$ \\
\hline$\Omega_{0}=2 \cdot 10^{11} \mathrm{~s}^{-1}$ & $\Omega_{0}=2 \cdot 10^{11} \mathrm{~s}^{-1}$ & $\Omega_{0} \sim 10^{11} \mathrm{~s}{ }^{-1}$ \\
$I_{\text {peak }}=100 \mathrm{GW} / \mathrm{cm}^{2}$ & $I_{\text {peak }}=100 \mathrm{GW} / \mathrm{cm}^{2}$ & $I_{\text {peak }}=20-30 \mathrm{GW} / \mathrm{cm}^{2}$ \\
$E_{\text {opt }}=110 \mathrm{~mJ}$ & $E_{\text {opt }}=11 \mathrm{~mJ}$ & $E_{\text {opt }} \sim 300 \mathrm{~mJ}$ \\
${ }^{a} \sim 6 \cdot 10^{-9}$ & $\eta \sim 10^{-11}$ & $\eta 10^{-8}$ \\
\hline \hline
\end{tabular}

${ }^{a}$ Efficiency is estimates as a ratio of the $\mathrm{THz}$ pulse energy to the total energy of optical pulses absorbed in the crystal $\eta=$ $E_{T H z} / 2 E_{\text {opt }}$. The ultimate efficiency of the method is given by the ratio of the $\mathrm{THz}$ radiation frequency to the frequency of the optical transitions $29 \mathrm{~cm}^{-1} / 14420 \mathrm{~cm}^{-1}=2 \cdot 10^{-3}$.

TABLE II: Estimates of the THz pulse energy, peak THz field amplitude, required optical pulse parameters and efficiency of $\mathrm{THz}$ radiation generation in ruby at low temperatures

\begin{tabular}{cc}
\hline \hline \hline ultrashort pulses $(10 \mathrm{ps}-1 \mathrm{~ns})$ & long pulses $(1 \mathrm{~ns}-10 \mathrm{~ns})$ \\
\hline$\sigma_{c b}=0.5$ & $\sigma_{c b}=1$ \\
$E_{T H z} \sim 7.5 \mu \mathrm{J}$ & $E_{T H z} \sim 8 \mu \mathrm{J}$ \\
$\mathcal{E}_{T H z} \sim 230 \mathrm{kV} / \mathrm{cm}$ & $\mathcal{E}_{T H z} \sim 170 \mathrm{kV} / \mathrm{cm}$ \\
\hline$\Omega_{0}=10^{10} \mathrm{~s}^{-1}$ & $\Omega_{0} \sim 5 \cdot 10^{9} \mathrm{~s}^{-1}$ \\
$I_{\text {peak }}=100 \mathrm{MW} / \mathrm{cm}^{2}$ & $I_{\text {peak }}=30 \mathrm{MW} / \mathrm{cm}^{2}$ \\
$E_{\text {opt }}=54 \mathrm{~mJ}$ & $E_{\text {opt }} \sim 270 \mathrm{~mJ}$ \\
$\eta \sim 10^{-4}$ & $\eta \sim 3 \cdot 10^{-5}$ \\
\hline \hline
\end{tabular}

\section{DISCUSSION}

\section{A. Application to other solid materials}

It is important to note that the proposed technique can be applied to other solid materials with suitable transitions in the $\mathrm{THz}$ range. Similar schemes can be found in other ions as well, since for a typical rare-earth or transition metal ion the level structure is complex, having many crystal field and spin-orbit split components, separated by tens to hundreds of wave numbers. For example, this method is readily applicable to $\mathrm{Cr}^{3+}: \mathrm{BeAl}_{2} \mathrm{O}_{4}$ (alexandrite), having R-lines splitting of $41 \mathrm{~cm}^{-1}(1.23 \mathrm{THz})$ in a non-inversion $\mathrm{Cr}^{3+}$ site [38], and R-lines absorption/emission cross-section ten times larger than ruby $\left(3 \cdot 10^{-19} \mathrm{~cm}^{2}\right.$ at room temperature). It allows one to decrease peak optical intensities to induce maximal $\mathrm{THz}$ coherence and results in higher convertion efficiency.
The technique can also be applied to rare-earth ion doped materials, for example $\mathrm{Pr}^{3+}$ doped hosts, such as $\mathrm{LaF}_{3}$ [39], $\mathrm{Y}_{2} \mathrm{SiO}_{5}$ [4], $\mathrm{CaF}_{2}$ [4] with the ground and excited state Stark splittings being in a broad range 17 - $100 \mathrm{~cm}^{-1}$ and higher, the same splittings are found in $\mathrm{Nd}^{3+}$ doped crystals, such as YAG [42] and $\mathrm{YVO}_{4}$ [4], and a number of others, for example Tm:YAG 44] and Er:YAG [4] and Er: $\mathrm{YLiF}_{4}$ [46].

Also it is important to note that the technique can be applied to gases as well, but the coupling to $\mathrm{THz}$ radiation in this case occurs by a magnetic-dipole moment (macroscopic polarization due to an electric-dipole moment is zero because of symmetry reasons). Typically the magnetic dipole moment is smaller than the electric dipole one, so efficiency is smaller, but for higher densities and pressures this approach can still produce $\mathrm{THz}$ radiation. The dipole moments for optical transitions can be stronger than for ruby, so the coherence can be larger. This possibility is an extension of the method proposed in [5, 6], although its analysis is beyond of the scope of the present paper.

The next important remark is related to the case when the $\mathrm{THz}$ transition of interest belongs to the ground state. Then there are a lot of new opportunities to use more sophisticated methods of coherence preparation. In particular, the method of pulsed coherence production via Stimulated Raman adiabatic passage (STIRAP) [22] can be used to control the duration of the $\mathrm{THz}$ pulse similarly to 6]. Generally, all methods for coherence preparation via femtosecond pulse shaping developed to improve the sensitivity of coherent Raman scattering [16], in particular, frequency chirping [16] and fractional STIRAP [15], 
matched pulses [21], etc. can be applied.

\section{B. Comparison with current methods of short $\mathrm{THz}$ pulse generation}

It is interesting to compare the method of $\mathrm{THz}$ generation proposed in recent works [5, 6] and in this paper with currently available ones. There are a variety of methods already considered successful for generation of short $\mathrm{THz}$ pulses, so it is worth to compare the above estimates with the currently achieved parameters.

At the moment the most impressive results in generation of short $\mathrm{THz}$ pulses were achieved using electron beam based sources, free-electron lasers (FEL) 47, 48] and synchrotrons [49, 50]. For FELs, typically THz pulses of about 1 ps duration with 1-40 ns distance between micropulses are generated, grouped into a few- $\mu$ s trains. Energies of the micropulses are about 1-50 $\mathrm{mJ}$ (the parameters of FELBE laser, Rossendorf, Germany). Recently, substantially higher powers of coherent broadband $\mathrm{THz}$ pulses, produced by synchrotron emission, were obtained from the electron beamline 49, 50]. Hundreds of fs-short half-cycle $\mathrm{THz}$ pulses were generated with energies up to $100 \mu \mathrm{J}$.

$\mathrm{THz}$ pulses with ns durations were achieved in $\mathrm{THz}$ semiconductor lasers (quantum cascade lasers, p-Ge and n-Si lasers [51]-[55]), limited by the need of cryogenic cooling and in the case of p-Ge lasers by high electric and magnetic fields required for laser operation. In p-Ge lasers [52]-54] 20 ps pulse durations were obtained in a mode-locked regime with peak power up to several Watts in a few- $\mu$ s train of pulses.

The obvious advantages of the optical crystal based $\mathrm{THz}$ emitters discussed in this work over FEL and synchrotron sources are compactness (typical optical solidstate materials are significantly smaller), cost, and ease of handling. Compared to $\mathrm{THz} \mathrm{QC}$ lasers the crystals are much easier to grow and handle, they also can provide better coupling between the $\mathrm{THz}$ mode and the generating material due to a larger size of crystals.

Other methods of short $\mathrm{THz}$ pulse generation are based on interaction of different materials with ultrashort laser pulses. The most popular ones, giving subpicosecond $\mathrm{THz}$ pulses, are the photocurrent method using the Auston-switch technique [56] and the optical rectification technique [57]. Since the proposed crystal-based $\mathrm{THz}$ sources are closer in their characteristics to these methods, we will make a more detailed comparison. Recently $\mathrm{THz}$ sources based on amlifier-laser systems such as Ti:Sapphire, utilizing different methods of THz radiation generation such as optical rectification in nonlinear crystals and laser-produced plasma and photocurrent $\mathrm{THz}$ generation in semiconductor antennas, became popular. The transition metal and rare-earth ion doped crystals proposed in this work as $\mathrm{THz}$ emitters are suitable for amplifier-laser-based $\mathrm{THz}$ systems working in a single-shot regime [58], since the typically long (hundreds $\mu \mathrm{s}$ - milliseconds) population decay time of excited states of these ions limits the repetion rate. Crystals are also better in terms of saturation, able to withstand high laser pulse fluence and thus very compatible with low-repetiotion rate table-top laser systems producing pulses with energies in the $10-100 \mathrm{~mJ}$ range with $\sim 10 \mathrm{~Hz}$ repetion rates. Fig 4 compares the predicted in [58] performance of different amplifier-laser-based $\mathrm{THz}$ sources and ruby at room temperature, at optical pulses energy of $\sim 10 \mathrm{~mJ}$. It shows that ruby is expected to perform as good as the biased GaAs antenna with $1 \mathrm{kV} / \mathrm{cm}$ dc bias field and the plasma $\mathrm{THz}$ source with an external bias field, slightly yielding them in conversion efficiency.

Fig 5 compares the predicted in $[58]$ performance of different amplifier-laser-based $\mathrm{THz}$ sources at room temperature and ruby at liquid helium one, at optical pulses energy of $\sim 100 \mathrm{~mJ}$. The ruby source at liquid helium temperature can compete with the the plasma source utilizing the fundumental and second harmonic optical fields in terms of $\mathrm{THz}$ pulse energy and conversion efficiency, and yields it in peak $\mathrm{THz}$ field amplitude.

\section{Advantages of the technique}

New methods of $\mathrm{THz}$ generation proposed in [4, 5,6$]$ for atomic and molecular gases and in the present work for doped crystals potentially have very high efficiency, they offer the possibility of $\mathrm{THz}$ pulses with controlled durations including femtosecond region and the possibility of generation of pulses with high energy, already comparable with synchrotron or free-electron laser based $\mathrm{THz}$ sources. Implementation of the method, therefore, will open exciting opportunities in many fields of $\mathrm{THz}$ applications such as the time-resolved $\mathrm{THz}$ spectroscopy. Using intense short pulses of $\mathrm{THz}$ radiation generated by the proposed method will significantly increase the performance of spectroscopic measurements, allowing maximal (among currently existing methods) temporal resolution. Extremely high efficiency of the proposed technique opens a way for orders of magnitude increase in sensitivity of the spectroscopic methods, $\mathrm{THz}$ tomogra- 
phy, non-destructive quality control, medical diagnostics and biomaterial characterization.

Another advantage of the proposed scheme is a better control over the phase of the generated $\mathrm{THz}$ radiation, resulting in lower (pulse duration $\times$ bandwidth) product compared to the non-coherent techniques. Resulting $\mathrm{THz}$ pulses have narrower bandwidth, thus offering an opportunity of better resolution measurements. Coherence of the generated $\mathrm{THz}$ pulses combined with their high energy opens the way to various non-linear phenomena in the $\mathrm{THz}$ range, as well as to coherent phenomena similar to the ones observed with lasers in the visible range, such as Rabi oscillations, coherent transients, etc. The proposed methods have, therefore, high potential for investigation of nonlinear effects in chemical and biological objects, in medical applications, which is a substantial, practically unexplored field of research.

\section{CONCLUSION}

In the present work we proposed a new technique for generation of short intense $\mathrm{THz}$ pulses in coherently driven doped optical crystals. The method is based on excitation of maximal $\mathrm{THz}$ coherence by a pair of resonant optical pulses, resulting in polarization build-up in the medium, which will radiate a $\mathrm{THz}$ pulse. As an ex- ample, a well-known laser material $\mathrm{Cr}^{3+}: \mathrm{Al}_{2} \mathrm{O}_{3}$ (ruby) is considered and numerical estimates of expected $\mathrm{THz}$ and required optical field parameters are given. A number of other doped crystals, having transitions in the $\mathrm{THz}$ range, are suggested. Comparison is made with the existing methods of short $\mathrm{THz}$ pulses generation.

In summary, the optical crystal based THz sources proposed in this work can potentially produce short high energy (hundreds pJ - tens $\mu \mathrm{J}$ ) $\mathrm{THz}$ pulses with durations ranging from 1 ps up to several ns. The generated $\mathrm{THz}$ radiation will be coherent, thus offering the possibility of coherent interaction between the $\mathrm{THz}$ pulses and probed media. This allows one to study various types of nonlinear penomena. Obvious advantages of crystals are their compact size, ease of growth and handling, robustness and low cost.

\section{ACKNOWLEDGEMENTS}

We gratefully acknowledge the support from the Air Force Office of Scientific Research, the National Science Foundation, the Defense Advanced Research Projects, the Office of Naval Research under Award No. N0001403-1-0385, and the Robert A. Welch Foundation (Grant No. A1261).
[1] Terahertz optoelectronics, edited by K. Sakai (Springer, Berlin, 2005); B.Ferguson, and X.-C.Zhang, Nature Materials 1, 26 (2002).

[2] Sensing with terahertz radiation, edited by D.Mittleman (Springer, New York, 2003).

[3] N.G.Kalugin, in Handbook of semiconductor nanostructures and nanodevices, edited by A.A.Balandin and K.L.Wang (American Scientific Publishers, Los Angeles, 2005).

[4] A. S. Zibrov, M. D. Lukin, L. Hollberg, and M. O. Scully, Phys. Rev. A 65, 051801(R) (2002).

[5] N.G.Kalugin, Y.Rostovtsev, M.O.Scully, Proc. SPIE 6120, 612002 (2006); N.G.Kalugin, Y.Rostovtsev, M.O.Scully, quant-phys/0602142 (2006).

[6] N.G.Kalugin, Y.Rostovtsev, Opt. Lett. 31, 969 (2006).

[7] O. Kocharovskaya and Ya.I. Khanin, Pis'ma Zn. Eksp. Teor. Fiz. 48, 581 (1988), (Sov. Phys. JETP Lett. 48, 630 (1988)); S.E. Harris, Phys. Rev. Lett. 62, 1033 (1989); M.O. Scully, S.Y. Zhu, and A. Gavrielides, Phys. Rev. Lett. 62, 2813 (1989).

[8] E. Arimondo in Progress in Optics $X X X V$, ed. by E. Wolf
(Elsevier, Amsterdam, 1996), p.257; S.E.Harris, Phys. Today 50, 36 (1997).

[9] A.S. Zibrov, M.D.Lukin, D.E.Nikonov, L.Hollberg, M.O.Scully, V.L.Velichansky, H.G.Robinson, Phys. Rev. Lett. 75, 1499 (1995); G.G. Padmabandu, G.R.Welch, I.N.Shubin, E.S.Fry, D.E.Nikonov, M.D.Lukin, M.O.Scully, Phys. Rev. Lett. 76, 2053 (1996).

[10] G.Alzetta, A.Gozzini, L.Moi, G.Orriols, Nuovo Cimento B 36, 5 (1976); H.R.Gray, R.M.Whitley, C.R.Stroud, Jr., Opt. Lett. 3, 218 (1978); K.J.Boller, A.Imamoglu, S.E.Harris, Phys. Rev. Lett. 66, 2593 (1991); J.E.Field, K.H.Hahn, S.E.Harris, Phys. Rev. Lett. 67, 3062 (1991).

[11] S.E.Harris, M.Jain, Opt. Lett. 22, 636 (1997).

[12] R.W.Boyd, M.O.Scully, Appl. Phys. Lett. 77, 3559 (2000).

[13] M.Jain, H.Xia, G.Y.Yin, A.J.Merriam, S.E.Harris, Phys. Rev. Lett. 77, 4326 (1996).

[14] O. Kocharovskaya, R. Kolesov, and Yu. Rostovtsev, Phys. Rev. Lett. 82, 3593-3596 (1999)

[15] V. A. Sautenkov, C. Y. Ye, Y. V. Rostovtsev, G. R. 
Welch, and M. O. Scully, Phys. Rev. A 70, 033406 (2004).

[16] M.O.Scully, G.W.Kattawar, P.R.Lucht, T.Opatrny, H.Pilloff, A.Rebane, A.V.Sokolov, M.S.Zubairy, Proc. Natl. Acad. Sci. U.S.A. 9, 10994 (2002).

[17] E. Kuznetsova, O. Kocharovskaya, P. Hemmer, and M. O. Scully, Phys. Rev. A 66, 063802 (2002).

[18] E. Kuznetsova, R. Kolesov, and O. Kocharovskaya, Phys. Rev. A 70, 043801 (2004).

[19] R.Kolesov, E. Kuznetsova, and O. Kocharovskaya, Phys. Rev. A 71, 043815 (2005).

[20] M.D. Frogley, J.F. Dynes, M. Beck, J. Faist, C.C. Phillips, Nature Mat. 5175 (2006).

[21] G. Beadie, Z.E. Sariyanni, Y.V. Rostovtsev, T. Opatrny, J. Reintjes, M.O. Scully, Opt. Commun. 244, 423 (2005).

[22] K. Bergmann, H. Theuer, and B. W. Shore, Rev. Mod. Phys. 70, 1003 (1998).

[23] D.S.Pestov, A.A.Belyanin, V.V.Kocharovsky, Vl.V.Kocharovsky, M.O.Scully, J. Mod. Opt. 51, 2523 (2004).

[24] M.O.Schweika-Kresimon, J.Gutschank, D.Suter, Phys. Rev. A 66, 043816 (2002).

[25] R.G.Brewer, R.L.Shoemaker, Phys. Rev. A 6, 2001 (1972).

[26] D.F.Nelson, and M.D.Sturge, Phys. Rev. 137, A1117 (1965).

[27] H.Lengfellner, K.F.Renk, IEEE J. Quant. Elect. 13, 421 (1977).

[28] A.A.Kaplyanskii and S.A.Basun, in Nonequilibrium Phonons in Nonmetallic Crystals, edited by W.Eisenmenger and A.A.Kaplyanskii (North-Holland, Amsterdam, 1986), p.373.

[29] N.M.Lawandy, IEEE J. Quant. Elect. 15, 401 (1979).

[30] D.W.Faries, P.L.Richards, Y.R.Shen, K.H.Yang, Phys. Rev. A 3, 3 (1971).

[31] T.Yajima, K.Inoue, Phys. Lett. A 26, 281 (1968).

[32] B.Halperin, J.A.Koningstein, D.Nicollin, Chem. Phys. Lett. 68, 58 (1979).

[33] S.A.Belozerov, G.M.Zverev, V.S.Naumov, V.A.Pashkov, Soviet Physics JETP 35, 158 (1972).

[34] G.M.Zverev, T.N.Mikhailova, V.A.Pashkov, N.M.Solov'eva, Soviet Physics JETP 26, 1053 (1968).

[35] N.Retzer, H.Lengfellner, and K.F.Renk, Phys. Lett. 96A, 487 (1983).

[36] D.E.McCumber, M.D.Sturge, J. Appl. Phys. 34, 1682 (1963).

[37] H.Lengfellner, J.Hummel, H.Netter, K.F.Rank, Opt. Lett. 8, 220 (1983).

[38] J.C.Walling, O.G.Peterson, H.P.Jenssen, R.C.Morris, E.W.O'Dell, IEEE J. Quant. Electr. 16, 1302 (1980).

[39] R.M.Shelby, R.M.Macfarlane, C.S.Yannoni, Phys. Rev. B 21, 5004 (1980).

[40] R.W.Equall, R.L.Cone, R.M.Macfarlane, Phys. Rev. B 52, 3963 (1995).

[41] R.M.Macfarlane, D.P.Burum, R.M.Shelby, Phys. Rev. B 29, 2390 (1984). a)

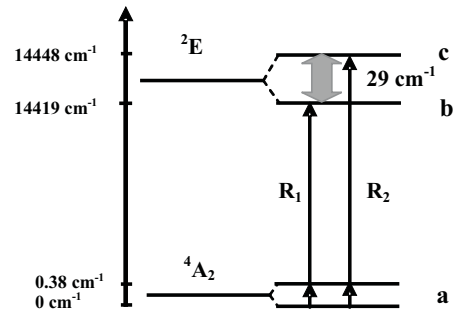

b)

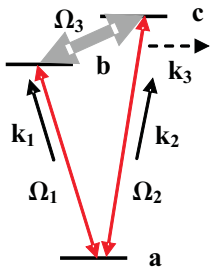

FIG. 1: a) Three-level $V$ energy system in ruby proposed for generation of $29 \mathrm{~cm}^{-1} \mathrm{THz}$ pulses; b) Model $V$ system of energy levels with two co-propagating fields 1 and 2 inducing coherence between levels $b$ and $c$.

[42] G.W.Burdick, C.K.Jayasankar, F.S.Richardson, M.F.Reid, Phys. Rev. B 50, 16309 (1994).

[43] D.K.Sandar, R.M.Yow, Opt. Mater. 14, 5 (2000).

[44] R.M.Macfarlane, J. of Lumin. 85, 181 (2000).

[45] J.B.Gruber, J.R.Quagliano, M.F.Reid, F.S.Richardson, M.E.Hills, M.D.Seltzer, S.B.Stevens, C.A.Morrison, T.H.Allik, Phys. Rev. B 48, 15561 (1993).

[46] R.A.Macfarlane, J. Opt. Soc. Am. B 8, 2009 (1991).

[47] W.Chin, et al., J. Phys. Chem. 122, 054317 (2005).

[48] X.G.Peralta, et al., Appl. Phys. Lett. 81, 1627 (2002).

[49] C.R.Neil, et al., Nucl. Instr. Meth. Phys. Res. A 507, 537 (2003).

[50] G.L.Carr, et al., J. Biol. Phys. 29, 319 (2003).

[51] R.Koehler, A.Tredicucci, F.Bertram, H.E.Beere, E.H.Linfield, A.G.Davies, D.A.Ritchie, R.C.Iotti, R.F.Rossi, Nature 417, 156 (2002).

[52] A.A.Andronov, I.V.Zverev, V.A.Kozlov, Yu.N.Nozdrin, S.A.Pavlov, V.N.Shastin, JETP Lett. 40, 804 (1984); E.Gornik, A.A.Andronov (ed), Opt. Quant. Electron. 23, S111 (1991).

[53] V.I.Gavrilenko, N.G.Kalugin, Z.E.Krasilnik, V.V.Nikonorov, A.V.Galyagin, P.N.Tsereteli, Semicond. Sci. Technol. 7, B649 (1992).

[54] A.V.Muravjov, R.C.Strijbos, C.J.Fredricksen, H.Weidner, W.Trimble, S.G.Pavlov, V.N.Shastin, R.E.Peale, Appl. Phys. Lett. 73, 3037 (1998).

[55] S.G.Pavlov, R.K.Zhukavin, E.E.Orlova, V.N.Shastin, A.V.Kirsanov, H.W.Hubers, K.Auen, H.Riemann, Phys. Rev. Lett. 84, 5220 (2000).

[56] X.-C.Zhang, B.B.Hu, J.T.Darrow, D.H.Auston, Appl. Phys. Lett. 56, 1011 (1990); D.H.Auston, K.P.Cheung, P.R.Smith, Appl. Phys. Lett. 45, 284 (1984).

[57] K.H.Yang, P.L.Richards, Y.R.Shen, Appl. Phys. Lett. 19, 320, (1971); A.Rice, X.F.Ma, X.-C.Zhang, D.Bliss, J.Larkin, M.Alexander, Appl. Phys. Lett. 64, 1324 (1994).

[58] T.Löffer, M.Kreb, M.Thomson, T.Hahn, N.Hasegawa, H.G.Roskos, Semicond. Sci. Technol. 20, S134 (2005). 


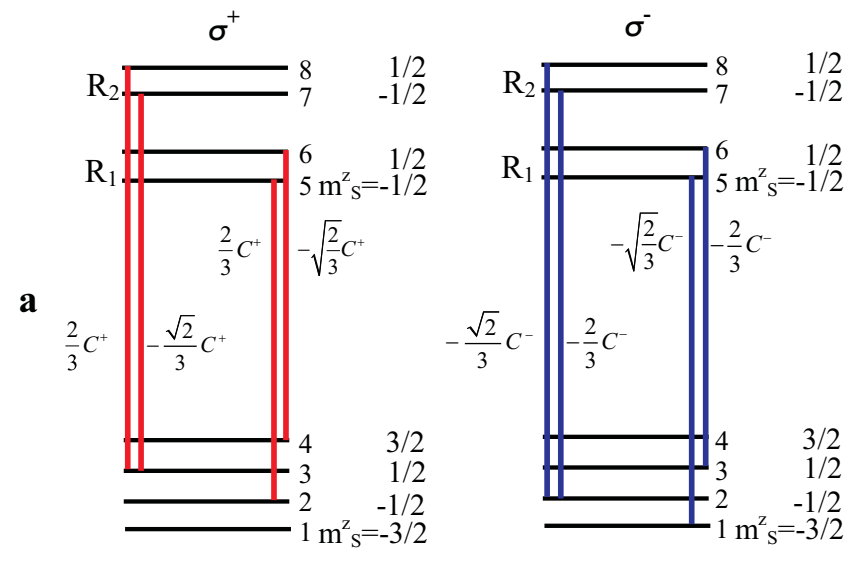

b
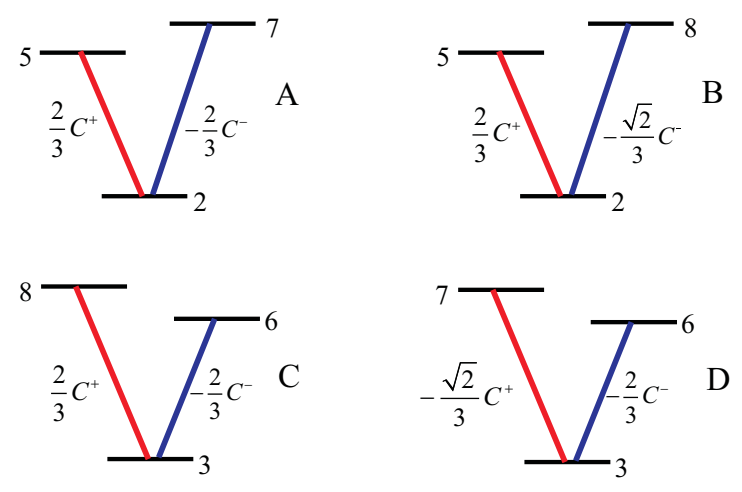

FIG. 2: a) Selection rules and transition matrix elements for $\mathrm{R}_{1}$ and $\mathrm{R}_{2}$ optical transitions in ruby for right and left circular polarizations; b) Four $V$ schemes possible for linearly polarized fields.

a)

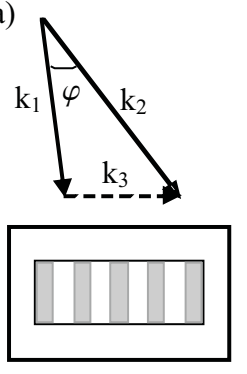

b)

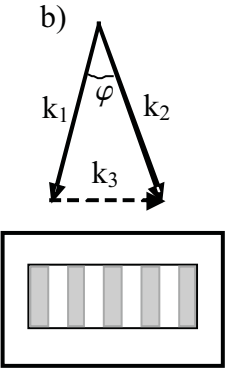

c)

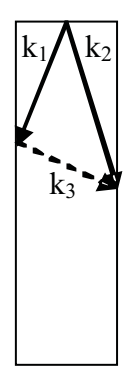

FIG. 3: a) CW fields 1 and 2 induce coherence between levels $b$ and $c$ in ruby; b) For short pulses $k_{1}=k_{2}$; c) A three mode ruby laser that generates optical radiation at $R_{1}$ and $R_{2}$ lines and $\mathrm{THz}$ radiation simultaneouly with the ruby crystal serving as a $\mathrm{THz}$ waveguide. 

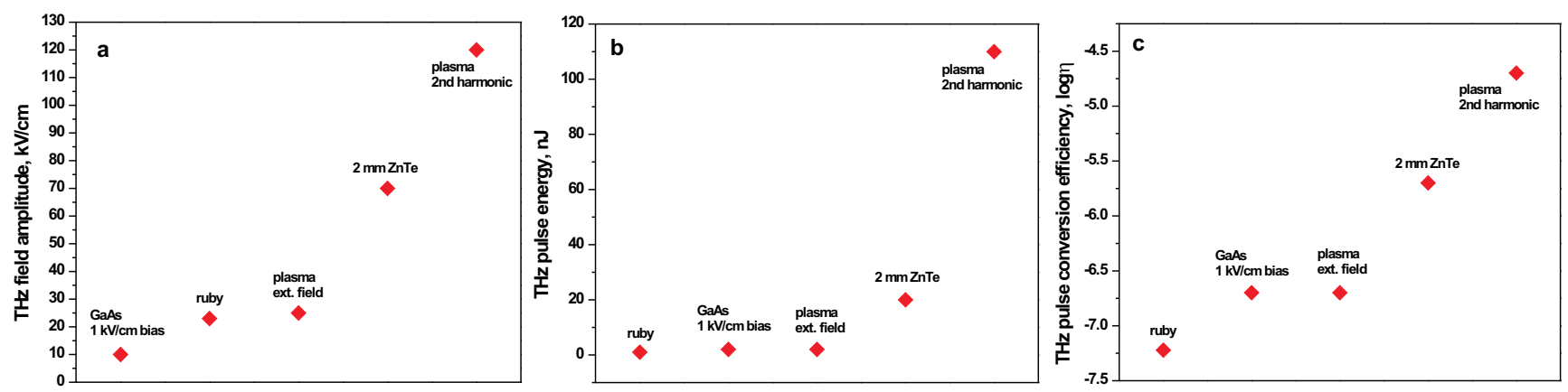

FIG. 4: Predicted in [58] performance of several amplifier-laser-based THz sources and ruby at room temperature with optical pulses energy $\sim 10 \mathrm{~mJ}$ : a) peak THz field amplitude; b) THz pulse energy; c) conversion efficiency.
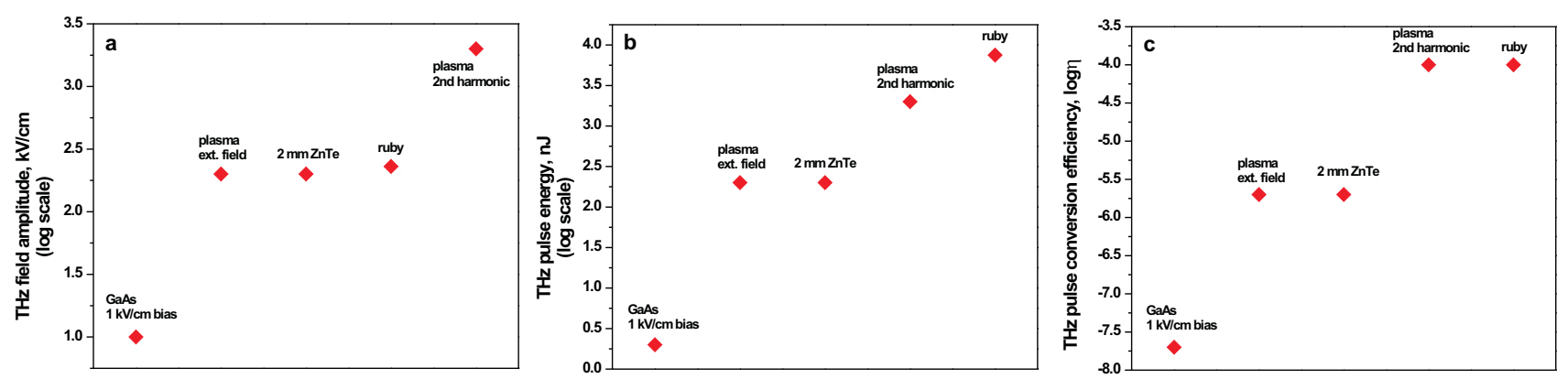

FIG. 5: Predicted in [58] performance of several amplifier-laser-based THz sources at room temperature and ruby at liquid helium one with optical pulses energy $\sim 100 \mathrm{~mJ}$ : a) peak THz field amplitude; b) THz pulse energy; c) conversion efficiency. 